Monatsschrift für Geburtshülfe u. Gynäkologie 1911;33:I-VI

\title{
Contents, Vol. 33, 1911
}

\section{Inhalts -Verzeichnis.}

Seite

Original-Arbeiten.

A $m$ a $n \mathrm{n}$, J. A., Ueber den Ersatz der Vagina bei voll-

kommenem Defekt derselben 553

Bartel, Julius und Edmund Herrmann, Ueber

die weibliche Keimdrüse bei Anomalie der Konstitution.

(Hierzu Taf. I-II) 125

Basset, R., Ueber Vorfall der Nachgeburt 421

--, Ueber Chondrodystrophia foetalis. (Hierzu Taf. Ill) 563

Bosse, Bruno, Der Dämmerschlaf oder die Skopo-

laminmorphin-Mischnarkose in ihrer Anwendung bei

Entbindungen und Operationen 316

Brenner, M., Eine neue Methode zur operativen Heilung

primärer oder rezidiver Cystocelen 464

Dobbert, Th., Die vaginalen Methoden in der Gynäko-

logie 27

, Antwort auf die Entgegnung Prof, von Otts auf die

vorstehende Arbeit 617

Döderlein, A., Ueber extraperitonealen Kaiserschnitt

und Hebosteotomie 1

Esau, Paul, Ringförmiger Ausriß der Scheide intra

partum (Kolpaporrhexis et Exorrhexis) 22

v. Franqué, Otto, Beiträge zur Operation der Pro

lapse nebst kurzen Bemerkungen zur anatomischen

Aetiologie. (Hierzu Taf. IV-V) 571

Heidemann, M., Ueber Gewichtsschwankungen Neugeborener, mit besonderer Berücksichtigung der Resultate bei vierstündlichem Anlegen 168

Heidenhain, L., Prolaps und Retroversio 587

Heinsius, Fritz, Versuche zur Vaccinebehandlung

der weiblichen Gonorrhoe $\quad 426$

Hellendall, Hugo, Zur akuten Magendilatation . 44

Kirchberg, Franz, Massage und Gymnastik (Phy-

sikalische Therapie) in Schwangerschaft und Wochenbett 433

- IV -

Klein, Gustav, Sectio caesarea vaginalis als Methode

der künstlichen Fehl- und Frühgeburt 145

Koch, Kurt, Autogene oder ektogene Infektion ? . . 297 
Krecke, Alb., Die Behandlung der freien eitrigen

Peritonitis mit Kampheröl 473

Lamers, A. J. M., Ein Fall von Sepsis im Wochenbett

nach Abort durch Staphylococcus aureus haemolyticus 161

Littauer, Arthur, Die Punktion von Ovarial-

cysten nach den Grundsätzen der modernen Chirurgie 455

$\mathrm{M}$ a y e r , A., Ueber das Uteruskarzinom und die Ergebnisse

seiner Behandlung mit Totalexstirpation nach Wertheim 701

Mirabeau, S., Urologisch-gynäkologische Mitteilungen 193

Mohr,L., und R. Freund, Ueber die Bolle der 01-

säure bei der Eklampsie (Erwiderung auf die Arbeiten

von Polano und v. Graff) 757

011 , D m. v., Die vaginalen Methoden in der Gynäkologie 475

--, Zweite und letzte Antwort an Herrn Dobbert auf

seinen Artikel, ,Die vaginalen Methoden in der Gynäko

logie" 626

Pachner, Franz, Zur Inversio uteri puerperalis . . 140

Pankow, 0., Ueber die ovarielle Ursache uteriner

Blutungen 339

Peitmann, Heinr., Zur Technik der Badikal-

operation des primären Scheidenkrebses $\quad 56$

Pruska, H., Ueber das Frühaufstehen der Wöchnerinnen 331

Bosenstein, M., Ueber Missed abortion. Ein Beitrag

zur Aetiologie der dabei nicht seltenen schweren Blu

tungen (Hierzu Taf. VI) 764

Bosenthal, Theodor, Intrauterine in Pseudarthrose

geheilte Humerusfraktur und Anencephalus 184

Sachs, E., Einseitiger Mangel des Ovariums mit rudi-

mentärer Entwicklung der Tube bei normaler Ausbildung

des Uterus 135

Schauta, F., Die erweiterte vaginale Operation bei

Carcinoma colli uteri auf Grund zehnjähriger Erfahrung 680

Semon,M., Bakteriologische Blutuntersuchungen bei

Puerperalfieber $\quad 148$

S i p p e 1, A., Zur Aetiologie und operativen Behandlung

des Uterusprolapses 610

Winter, G., Die Bekämpfung des Krebses im Königreich

Preussen 754

$-\mathrm{V}-$

Yamasaki, M., Beitrag zur Aetiologie der Ovarial-

dermoide und zur Kenntnis der darin vorkommenden

Haare. Mitteilung eines Falles von Dermoid mit weißen

Haaren 63

Zöppritz, B., Ueber bakterizide Eigenschaften des

Vaginalsekrets und des Urins Schwangerer 276

Tagesfragen. 
Das Martyrium der heiligen Agathe im 20. Jahrhundert.

Von W. Bokelmann (Mit Kunstbeilage) 677

Ueber Ròntgentherapie. Von A. Döderlein 413

Tuberkulose und Schwangerschaft. Von F, S c h a u t a, . 265

Sammelreferate.

Tuberkulose und Schwangerschaft. Von Dr.KurtHenius

in Berlin $\quad 345$

Ueber Pathologie und Therapie der Eklampsie. Von Dr.

Adolf Hüffelin Darmstadt 199

Die operative Behandlung der umbilicalen und epigastrischen

Hernien. Von Dr. Martin Kirschner in

Königsberg i. Pr 211

Zur Anästhesierungsfrage in der Gynäkologie. Von Dr.

Sigmund Stiassny in Wien 77

Die dänische gynäkologische Literatur 1909. Von Dr.

Victor Albeck in Kopenhagen 493

Die geburtshülfliche und gynäkologische Literatur in

Holland 1909. II. Sem. Von Dr. M. M. de M o n c h y

in Haag 773

Polnische geburtshülfliche und gynäkologische Literatur 1909.

Von Dr. Hugo Ehrlich in Przemysl 216

Bericht über die geburtshülflich-gynäkologische Literatur in

Nord-Amerika. Januar-Juli 1909 (und teilweise No-

vember-Dezember 1908). Von Dr. Carl Eggers

in New York 497

Berchte.

Vom 40. Kongreß der Deutschen Gesellschaft für Chirurgie zu

Berlin vom 19.-22. April 1911. Von Priv.-Doz. Dr. W.

Sigwart in Berlin 777

$-\mathrm{VI}-$

Vom Kongreß für innere Medizin in Wiesbaden vom 19. bis

22. April 1911. Bericht, erstattet von Dr. Meyer -

B u t z 781

Gustav von Braun $\dagger .349$

Tagesordnung für die XIV. Versammlung der Deutschen

Geseilschaft für Chirurgie in München vom

7.-10. Juni $1911 \quad 787$

Tagesnachrichten und Personalien 94, 222, 351, 514, 629, 792

Vereins- und Literaturbeilage,

Gynäkologische Geseilschaft in Breslau 95, 231, 352, 515, 793

Geseilschaft für Gynäkologie und Geburtshülfe zu Köln . . 246

Mittelrheinische Geseilschaft für Geburtshülfe und Gynä

kologie $\quad 379,630, \quad 801$

Münchener Gynäkologische Geseilschaft . . 100, 239, 398, 647

Niederrheinisch-westfälische Geseilschaft für Gynäkologie

und Geburtshülfe

114 
Nordostdeutsche Geseilschaft für Gynäkologie .... 223,

Nordwestdeutsche Geseilschaft für Gynäkologie .... 365

Referate aus geburtshülflich-gynäkologischen Gesellschaften

$116,249,404,539,660$ 830

Geburtshülflich-gynäkologische Mitteilungen aus anderen Ge sellschaften und Vereinen . . 118, 250, 404, 540, 660,

Besprechung 405

Buchanzeigen 119, 251, 406, 540, 661

Literatur-Verzeichnis 120, 260, 409, 549, 674,

Saehregister

837

Namenregister

843 